\title{
Characteristics of the Drag Coefficient over a Tropical Environment in Convective Conditions*
}

\author{
PIYUSH SRIVASTAVA AND MAITHILI SHARAN \\ Centre for Atmospheric Sciences, Indian Institute of Technology Delhi, New Delhi, India
}

(Manuscript received 16 December 2014, in final form 20 May 2015)

\begin{abstract}
The turbulent data over a tropical region are utilized to analyze the observational behavior of the drag coefficient $C_{D}$ with respect to wind speed $U$ and the stability parameter $\zeta$ in convective conditions. The drag coefficient is observed to follow the power-law profile with respect to $U$, with large values in low winds and relatively lower values with moderate-wind conditions. Depending on the stability regimes, regression curves for $C_{D}$ with $U$ are proposed. The variation of $C_{D}$ with $\zeta$ is bounded by a curve. This curve first shows increasing behavior with $-\zeta$ until it reaches a peak at $\zeta \approx-0.12$ and then decreases with increasing instability. A mathematical analysis based on Monin-Obukhov similarity (MOS) reveals that $C_{D}$ increases monotonically with increasing instability. This suggests that MOS theory is able to capture the increasing nature of $C_{D}$ in weakly to moderately unstable conditions. However, it is unable to explain the observed decreasing behavior of $C_{D}$ with $-\zeta$ in moderately to strongly unstable conditions in the tropics within the framework of commonly used similarity functions.
\end{abstract}

\section{Introduction}

The transfer coefficient of momentum (surface drag coefficient) plays an important role in determining the momentum exchange process between land and atmosphere. The surface drag coefficient $C_{D}$ over land is normally computed using the Monin-Obukhov similarity (MOS) theory (Monin and Obukhov 1954) as a function of stability using the expression (Mahrt et al. 2001; Sharan and Kumar 2011)

$$
C_{D}=k^{2}\left\{\ln \left(z / z_{0}\right)-\psi_{m}\left[\zeta,\left(z_{0} / z\right) \zeta\right]\right\}^{-2},
$$

where $k$ is the von Kármán constant, $z$ is the height above the ground, $\zeta=z / L$ ( $L$ is the Obukhov length scale) is the stability parameter, $z_{0}$ is the roughness length of momentum, and $\psi_{m}$ is the integrated similarity function given by

\footnotetext{
* Supplemental information related to this paper is available at the Journals Online website: http://dx.doi.org/10.1175/JAS-D-140383.s1.

Corresponding author address: Prof. Maithili Sharan, Centre for Atmospheric Sciences, Indian Institute of Technology Delhi, Hauz Khas, New Delhi 110016, India.

E-mail: mathilis@cas.iitd.ernet.in
}

$$
\psi_{m}\left(\zeta, \frac{z_{0}}{z} \zeta\right)=\int_{\left(z_{0} / z\right) \zeta}^{\zeta} \frac{1-\varphi_{m}\left(\zeta^{\prime}\right)}{\zeta^{\prime}} d \zeta^{\prime},
$$

in which $\zeta^{\prime}$ is a dummy variable of integration corresponding to $\zeta$.

The similarity function $\varphi_{m}$ in unstable conditions is (Businger et al. 1971)

$$
\varphi_{m}(\zeta)=\left(1-\gamma_{m} \zeta\right)^{-1 / 4}
$$

where $\gamma_{m}$ is a constant.

A number of studies have been carried out to analyze the observational characteristics of $C_{D}$ over land and over sea surfaces, but a consistent agreement has not yet emerged. Rao et al. (1996) analyzed the Monsoon Trough Boundary Layer Experiment (MONTBLEX-90) dataset for both arid and moist regions and concluded that $C_{D}$ varies with wind speed according to a power law. Under unstable conditions at low winds, a substantial disagreement with MOS theory was observed (Rao et al. 1996). Mahrt et al. (2001) analyzed datasets over different types of surfaces and observed that $C_{D}$ increases with decreasing wind speed in all stability regimes. This was attributed to the enhanced viscous effects and reduction in the streamlining of the surface obstacles causing an increment in surface roughness length. Zhu and Furst (2013) argued that observed increasing behavior of $C_{D}$ 


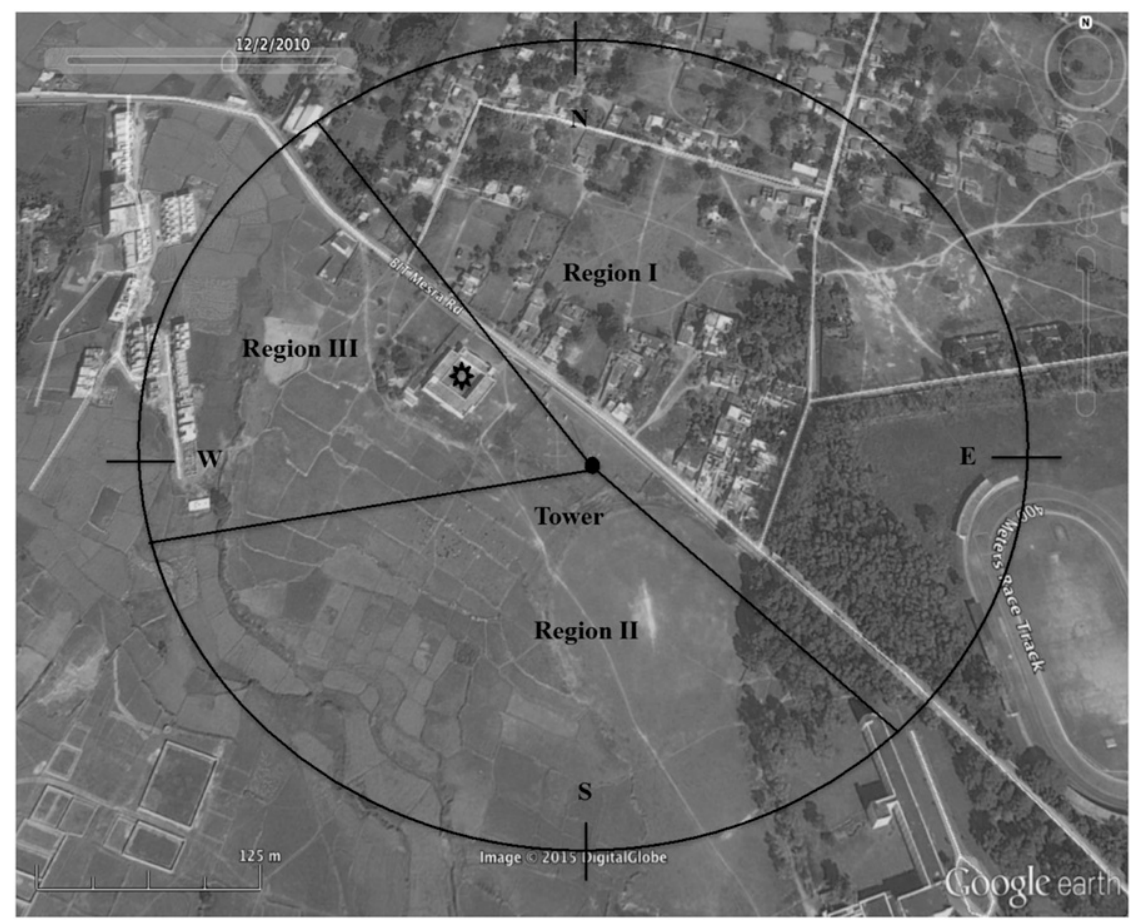

FIG. 1. The location of the tower (marked with a point) and the surrounding area from a Google map. Regions I, II, and III are classified in accordance with the land cover and separated by the lines. A school building near the tower is marked by an asterisk.

with decreasing wind speed could not be explained independently on the basis of change in $z_{0}$, as suggested by Mahrt et al. (2001). There is a sharp increase of $z_{0}$ as wind speed decreases, and it can reach up to $10 \mathrm{~m}$ for extremely low-wind speeds, which is not feasible for most of the overland conditions (Zhu and Furst 2013). A new parameterization scheme for the drag coefficient based on the turbulent kinetic energy budget (Zhu and Furst 2013) provides an explanation for the observed variations of $C_{D}$ with wind speed, particularly in low-wind conditions.

The studies done so far are primarily focused on analyzing the behavior of $C_{D}$ with the wind speed, and very limited studies have been carried out to analyze the observational variation of $C_{D}$ with the stability parameter (Mahrt et al. 2001; Niu et al. 2012; Furst 2013; Peng and Sun 2014). In stable conditions, variation of $C_{D}$ with $\zeta$ is observed to be consistent with the MOS theory, and $C_{D}$ is observed to decrease with increasing stability. However, the observational variation of $C_{D}$ with respect to $\zeta$ in unstable conditions has not been analyzed so far in a consistent manner. Further, this becomes complex with the weakening of winds. The MOS theory predicts that $C_{D}$ increases with increasing instability (appendix A). However, Mahrt et al. (2001) observed that $C_{D}$ does not depend systematically on the stability parameter for significantly unstable conditions. It has been found that $C_{D}$ does properly correlate with $\zeta$ in unstable conditions (Niu et al. 2012), and larger values of $C_{D}$ have been observed in near-neutral conditions, compared to significantly unstable conditions.

The understanding of $C_{D}$ assumes significance in the tropics, because deep convective mixing is a dominant feature of the tropical environment. Therefore, the objective of the present study is to analyze the observed nature of the drag coefficient in convective conditions over a tropical region.

\section{Data}

The data used in the present study are obtained from slow- and fast-response sensors mounted over a $32-\mathrm{m}$ micrometeorological tower deployed in a remote grassland area of Birla Institute of Technology, Mesra, Ranchi $\left(23.412^{\circ} \mathrm{N}, 85.440^{\circ} \mathrm{E}\right)$, India, with an average elevation of $609 \mathrm{~m}$ above mean sea level in a tropical region (Fig. 1). There are few suburban buildings in the area between the east and the northwest. There are hostel buildings, residential houses, and dense trees in the area between the southeast and the east (Tyagi et al. 2012; Dwivedi et al. 2014). A building nearest to the tower is a school to the northwest. There is agricultural land in the area between the northwest and the west. The area between the southeast and the west is relatively flat and free from any obstacle. 
TABLE 1. Quantitative description of the data in each of the unstable sublayers according to Bernardes and Dias (2010).

\begin{tabular}{|c|c|c|c|c|c|}
\hline \multirow[b]{2}{*}{ Sublayer } & \multirow[b]{2}{*}{ Range of $\zeta$} & \multicolumn{2}{|c|}{ Number of points } & \multicolumn{2}{|c|}{ Average value of $C_{D}$} \\
\hline & & Low & Moderate & Low & Moderate \\
\hline DNS & $-0.04 \leq \zeta \leq 0$ & 10 & 48 & 0.10058 & 0.02240 \\
\hline DNS-DCS transition & $-0.12 \leq \zeta<-0.04$ & 25 & 227 & 0.11366 & 0.02477 \\
\hline DCS & $-1.20 \leq \zeta<-0.12$ & 141 & 250 & 0.09030 & 0.01758 \\
\hline DCS-FCS transition & $-2.00 \leq \zeta<-1.20$ & 11 & 11 & 0.04030 & 0.00666 \\
\hline FCS & $\zeta<-2.0$ & 33 & 15 & 0.02410 & 0.00317 \\
\hline
\end{tabular}

The slow-response sensors at logarithmic heights of 1 , $2,4,8,16$, and $32 \mathrm{~m}$ on the tower measure air temperature, wind speed, wind direction, and relative humidity. A fast-response sensor (CSAT3 sonic anemometer) at $10-\mathrm{m}$ height measures the three components of wind and temperature at a $10-\mathrm{Hz}$ frequency (https://www.bitmesra. ac.in/cms-aboutus.aspx?this=1\&mid=16\&cid=17).

\section{Analysis and methodology}

This study analyzes turbulent measurements for 3 months (March-May 2009) corresponding to the premonsoon period taken at $10-\mathrm{m}$ height with a CSAT3 sonic anemometer. The approach of Vickers and Mahrt (1997) is adopted for removing the spikes present in the dataset. After the quality check, data were rotated into a streamline coordinate system. The mean diurnal behavior of the wind speed, temperature, and heat flux is given in Fig. S1 (see the electronic supplementary material). Frictional velocity $u_{*}$ is calculated from the expression

$$
u_{*}=\left[\left(\overline{u^{\prime} w^{\prime}}\right)^{2}+\left(\overline{v^{\prime} w^{\prime}}\right)^{2}\right]^{1 / 4}
$$

in which $u^{\prime}, v^{\prime}$, and $w^{\prime}$ are, respectively, the fluctuations in longitudinal, lateral, and vertical wind components.

The stability parameter is calculated from the expression

$$
\zeta=z / L=-\frac{k z g\left(\overline{w^{\prime} \theta_{v}^{\prime}}\right)}{\overline{\theta_{v}} u_{*}^{3}},
$$

where $\overline{\theta_{v}}$ is the mean virtual temperature in Kelvin, $g$ is acceleration due to gravity, and $\theta_{v}^{\prime}$ is fluctuation in the virtual temperature.

The drag coefficient is obtained as

$$
C_{D}=\left(u_{*} / U\right)^{2},
$$

where $U$ is the mean wind speed.

The whole dataset is divided based on the wind speed and the stability regimes. As the focus of the study is on unstable conditions, data during the daytime conditions with $\zeta<0$ are used. The data that correspond to wind speed less than $0.1 \mathrm{~m} \mathrm{~s}^{-1}$ and the transition period between day and night are excluded. To minimize the effect of the rainfall on the measurements, the data points corresponding to the $1 \mathrm{~h}$ before and after rainfall are also excluded. A total of 772 hourly data points with $\zeta<0$ are obtained with $0.1 \leq U \leq 8 \mathrm{~m} \mathrm{~s}^{-1}$, out of which 221 and $551 \mathrm{~h}$ correspond to low $\left(\leq 2 \mathrm{~m} \mathrm{~s}^{-1}\right)$ and moderate $\left(>2 \mathrm{~m} \mathrm{~s}^{-1}\right)$ winds, respectively. Since most of the data points corresponding to the range $U>2 \mathrm{~m} \mathrm{~s}^{-1}$ fall in the range $2<U \leq 6 \mathrm{~m} \mathrm{~s}^{-1}$ and only approximately $2 \%$ of data belong to the range $U \geq 6 \mathrm{~m} \mathrm{~s}^{-1}$, we refer to all the data points corresponding to $U>2 \mathrm{~m} \mathrm{~s}^{-1}$ as moderate-wind condition. The dataset is further classified into five unstable sublayers, as suggested by Kader and Yaglom (1990) and Bernardes and Dias (2010). These sublayers are dynamic (DNS), dynamic-dynamic convective transition (DNS-DCS transition), dynamic convective (DCS), dynamic convective-free convective transition (DNS-FCS transition), and free convective (FCS). The quantitative description of the data in each sublayer is given in Table 1. The data lying in the DNS and DNS-DCS transition layers $(-0.12 \leq \zeta<0)$ characterize weakly to moderately unstable conditions, while data that belong to the DCS, DCS-FCS transition, and FCS layers $(\zeta<-0.12)$ represent moderately to strongly convective conditions. Depending on the wind directions, the filtered data are divided into 24 sectors, each covering $15^{\circ}$, in order to give a better understanding of the effect of upwind surface type on the behavior of drag coefficient.

\section{Results and discussion} a. The observed behavior of $C_{D}$ with respect to wind
speed in different sublayers

An increasing nature of $C_{D}$ is observed (Fig. 2) with a decrease in the wind speed. From Eq. (6), $C_{D}$ increases with increasing $u_{*}$ whereas it decreases with an increase in $U$. Despite these opposing tendencies, $C_{D}$ increases with a decrease in wind speed because the rate of increase of $C_{D}$ with $u_{*}$ is relatively smaller than the corresponding rate of decrease with $U$.

The regression curves for this dataset are found to be powerlaw profiles: (i) $C_{D}=0.17 U^{-1.57}$ (DNS), (ii) $C_{D}=0.10 U^{-1.50}$ (DNS-DCS transition), (iii) $C_{D}=0.10 U^{-1.47}$ (DCS), (iv) 

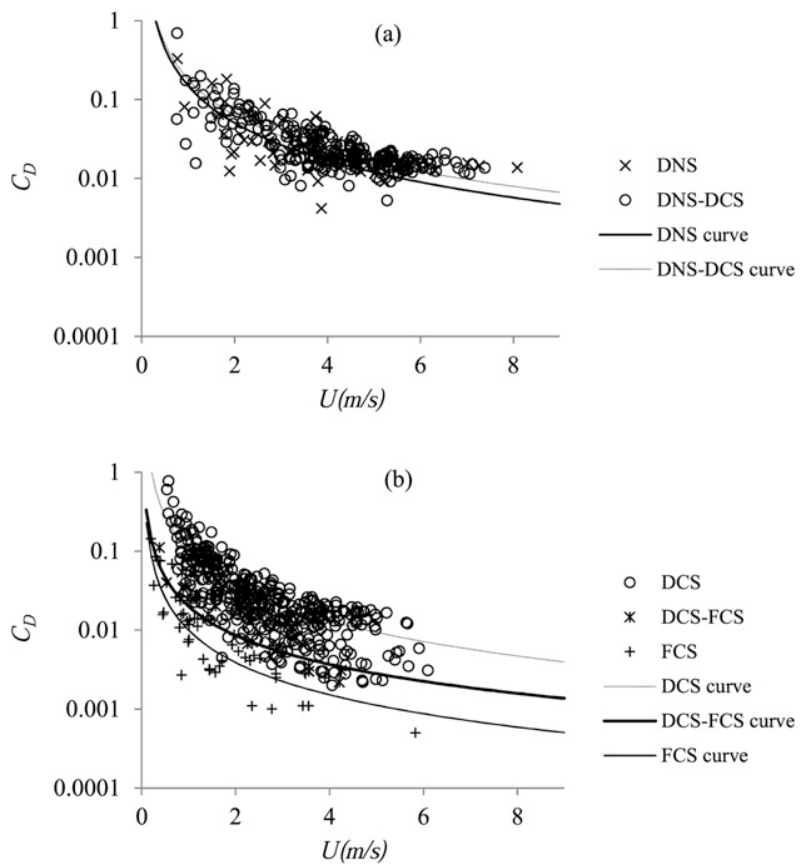

○ DCS

* DCS-FCS

+ FCS

DCS curve

DCS-FCS curve

- FCS curve

FIG. 2. Variation of $C_{D}$ with respect to $U$ in unstable conditions during March-May 2009 for (a) DNS and DNS-DCS and (b) DCS, DNS-FCS, and FCS. The solid line in each panel represents the best-fit curve for each sublayer.

$C_{D}=0.02 U^{-1.12}$ (DCS-FCS transition), and (v) $C_{D}=$ $0.01 U^{-1.36}$ (FCS). Rao et al. (1996) also observed the variation of $C_{D}$ with $U$ as a power-law profile: $C_{D}=0.04 U^{-1.06}$ at Jodhpur station in MONTBLEX-90. The average values of $C_{D}$ are observed to be 0.08 and 0.02 in low and moderate winds, respectively. These values are relatively higher compared to those reported by Rao et al. (1996) over Jodhpur. However, the drag in low wind was reported as an order of magnitude higher than that in moderate to strong winds. In contrast to weakly to moderately unstable conditions (DNS and DNS-DCS transition layers) (Fig. 2a), there is a relatively large scattering (Fig. 2b) of $C_{D}$ with $U$ in moderate to strong instability (DCS, DCS-FCS transition, and FCS layers). This indicates that the correlation of $C_{D}$ is relatively better with $U$ in weakly to moderately unstable conditions, suggesting that the relationship appears to be significantly influenced by the stability of the atmospheric surface layer. However, it is not feasible to isolate the effects of instability and the wind speed on the values of drag coefficient because of self-correlation (Mahrt et al. 2001).

\section{b. The observed behavior of $C_{D}$ with respect to $U$ in different wind direction sectors}

Land-based observations of $C_{D}$ are affected by the upwind land types; hence, we analyze the behavior of

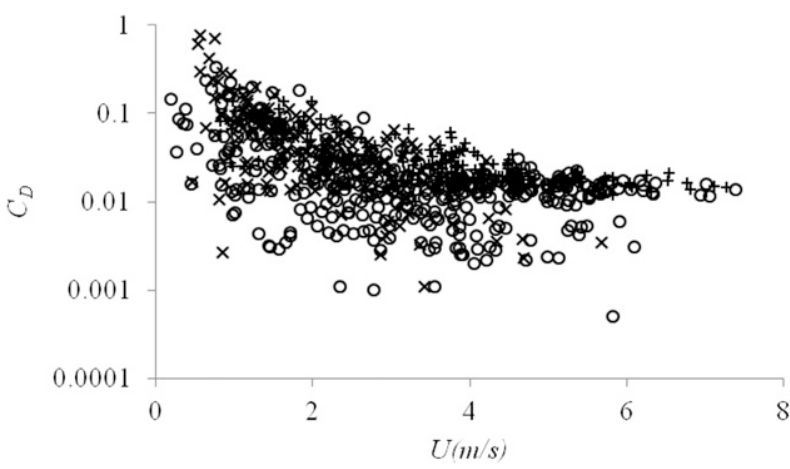

FIG. 3. Variation of $C_{D}$ with respect to $U$ in unstable conditions during March-May 2009. The symbols $\times, \bigcirc$, and + correspond to the data points falling in the regions I, II, and III, respectively.

$C_{D}$ with respect to $U$ in different wind-direction sectors. The wind direction is taken as a clockwise angle from the north and is divided into 24 sectors with an interval of $15^{\circ}$ and numbered accordingly as $1,2, \ldots$, and 24. There are some buildings and trees in sectors 1-9 and 23 and 24 (denoted as region I) while there is agricultural land lying in sectors 17-22 (region III). Sectors 10-16 (denoted as region II) belong to a relatively flat area. The observed behavior of $C_{D}$ with $U$ remains almost similar in each of the regions following the power law (Fig. 3). The average value of $C_{D}$ is observed to be relatively large in region $\mathrm{I}(\sim 0.069)$ compared to region II $(\sim 0.026)$ and region III $(\sim 0.033)$. This may be attributed to the roughness elements and a relatively high frequency of occurrence of low-wind conditions in region I. The average value of $C_{D}$ in region III is observed to be slightly higher compared to that in region II, which may be a result of the presence of agricultural land and a school building near the tower in region III.

\section{c. The observed behavior of $C_{D}$ with $\zeta$}

The scatter in $C_{D}$ with $\zeta$ is relatively large in low-wind (Fig. 5a) compared to moderate-wind (Fig. 4a) conditions. Though the observed values of $C_{D}$ reveal no systematic dependence on $\zeta$ in weak to moderate instability (DNS and DNS-DCS transition layers), it shows a relatively better correlation with wind speed (Fig. 2a) in this stability regime. This is consistent with the observations of Mahrt et al. (2001), which suggest that drag coefficient appears to be more related to $U$ than $\zeta$ for moderately and very unstable conditions.

The variation of drag coefficient with $\zeta$ is bounded by a curve. This curve first shows increasing behavior with $-\zeta$ until it attains a peak at $\zeta \approx-0.12$ and then decreases with increasing instability. This behavior is observed for both low- and moderate-wind conditions. A large scatter is observed (Figs. 4a and 5a) near the point of transition between 
(a)

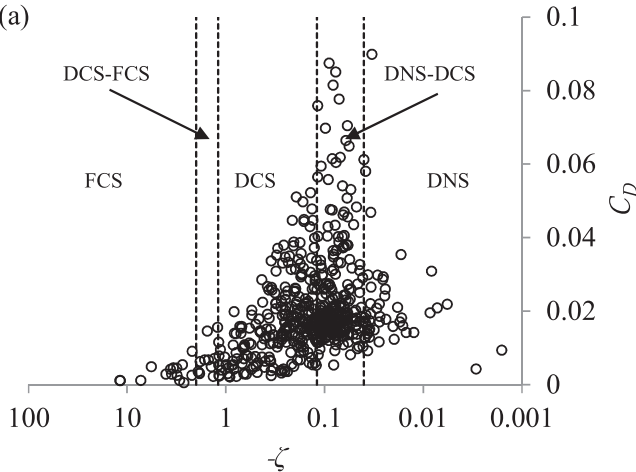

(b)

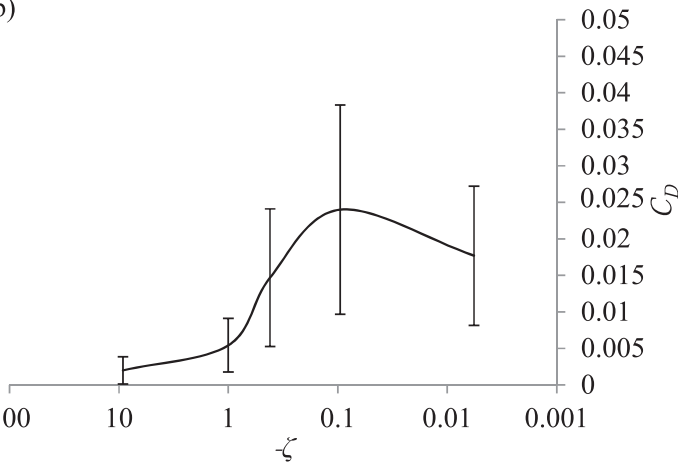

(c)

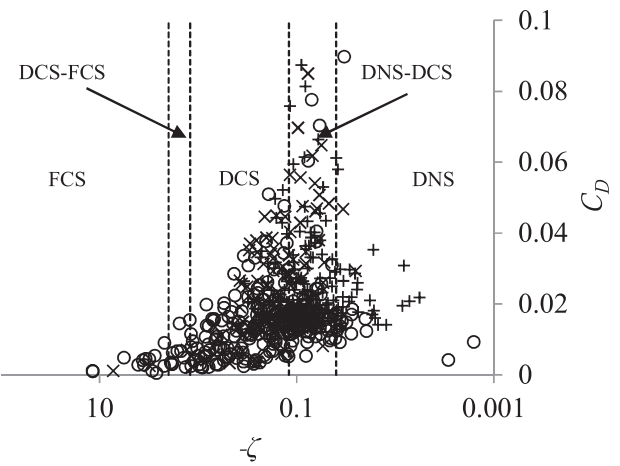

FIG. 4. Variation of $C_{D}$ with $\zeta$ in moderate-wind unstable conditions during March-May 2009. (a) A combined scatterplot for all the wind directions; (b) a corresponding bin diagram; and (c) a scatterplot in different regions. In (c), the symbols $\times, \bigcirc$, and + correspond to the data points falling in the regions I, II, and III, respectively. In (a) and (c), the vertical dashed lines separate the different sublayers.

DNS-DCS and DCS layers (i.e., $\zeta \approx-0.12$ ) in both lowand moderate-wind conditions. The bin diagram shows that $C_{D}$ increases with increasing instability in DNS and DNS-DCS transition layers in moderate-wind conditions (Fig. 4b), but this relationship is not very clear in case of low winds (Fig. 5b) because there are fewer data points in DNS. However, an increasing nature of $C_{D}$ with $-\zeta$ in the DNS-DCS transition layer is observed (Fig. 5b) in low-wind conditions from the bin diagram. A decreasing trend of $C_{D}$ is observed (Figs. $4 \mathrm{a}$ and $5 \mathrm{a}$ ) from its peak value with increasing instability in both low- and moderate-wind regimes.

Table 1 shows the amount of data and the observed average values of $C_{D}$ in each of the sublayers. There is an increase in the average values of $C_{D}$ from the DNS to the DNS-DCS layer, and it starts decreasing significantly from the DCS to the FCS layers. Thus, from Figs. 4a and 5 a and Table 1 , we conclude that $C_{D}$ increases in weak to moderate instability, reaching a peak value, beyond which it starts decreasing with increasing instability.

A physical reasoning for the observed decreasing nature of $C_{D}$ from its peak value with increasing instability is not clear to us at this moment. However, it may be explained partly in light of the definition of $C_{D}$ in terms of $u_{*}$ and $U$. The frictional velocity increases with $-\zeta$ (Figs. 6a and 6b) in DNS and DNS-DCS transition layers. However, a large scatter is observed in $u_{*}$ with $-\zeta$ in these sublayers, resulting in a large scattering in $C_{D}$ (Figs. 4a and 5a) in both low- and moderate-wind conditions. The values of $u_{*}$ are observed to decrease from its peak value in the DNS-DCS to FCS layers in both the wind regimes (Figs. $6 a$ and $6 \mathrm{~b}$ ) implying that the wind shear is observed to decrease with increasing instability. Thus, it appears that the increase in the turbulence in the DCS to FCS layers is primarily associated with convective motions, rather than mechanical motions, resulting in weak wind shear along with the strong instability. The conclusions drawn here are consistent with the observation of Yusup (2012), in which small values of $u_{*}$ are observed in very unstable as well as stable conditions.

The behavior of $C_{D}$ with $-\zeta$ is analyzed in different regions associated with the wind direction variations, and it is observed that the decreasing nature of $C_{D}$ with $-\zeta$ from its peak value persists in each of the regions (Figs. $4 \mathrm{c}$ and $5 \mathrm{c}$ ). However, the nature is relatively more pronounced in region II compared to regions III and I.

The analysis of the limited turbulent measurements for April and May 1999 at Anand $\left(22^{\circ} 35^{\prime} \mathrm{N}, 72^{\circ} 55^{\prime} \mathrm{E}\right)$ 

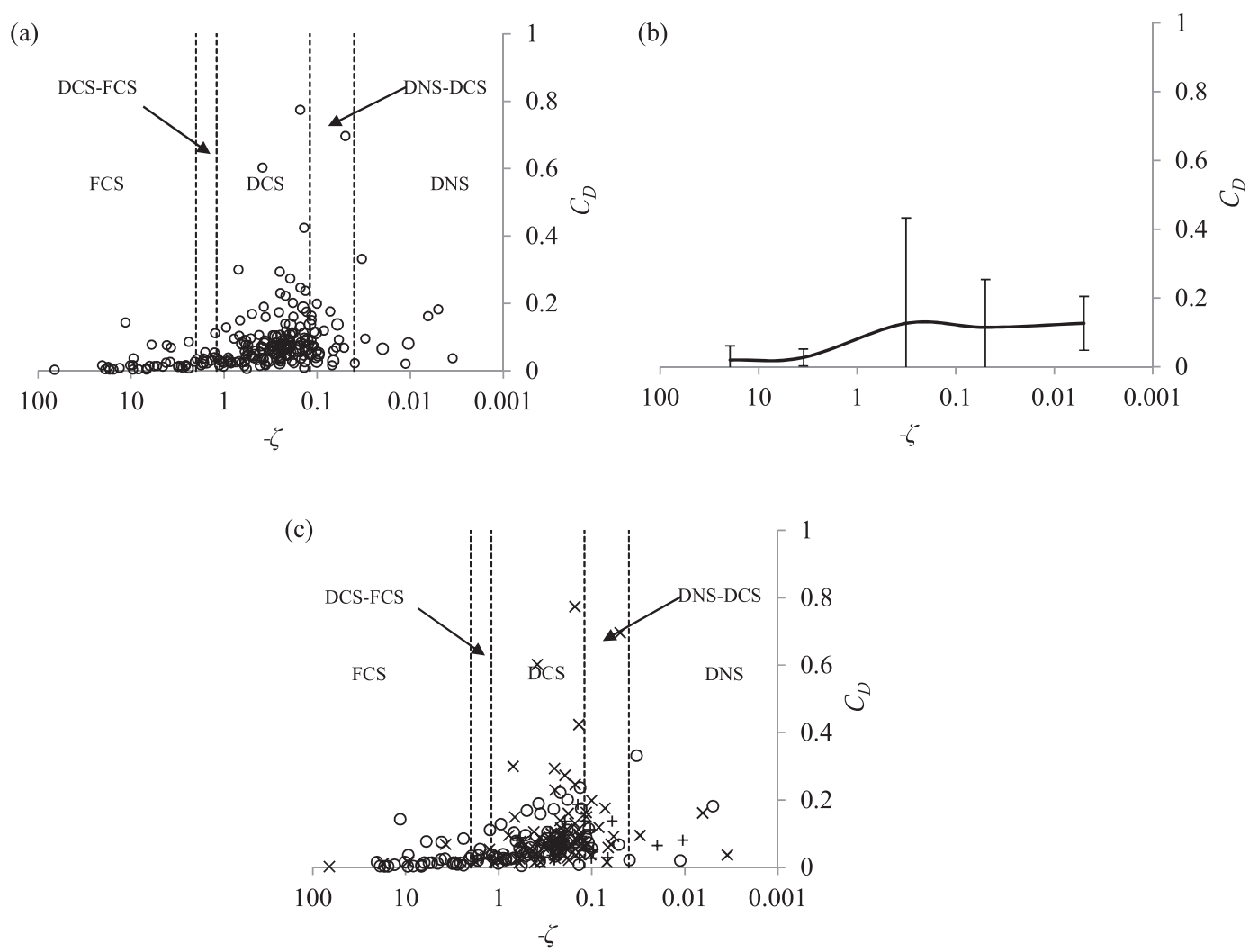

FIG. 5. As in Fig. 4, but for the low-wind conditions.

during the Land Surface and Processes Experiment (LASPEX) also reveals a decreasing tendency of $C_{D}$ from its peak value with increasing instability (not shown here). By analyzing the data for selected days of LASPEX, Patil (2006) observed a high magnitude of $C_{D}$ for the weakly unstable conditions. Furst (2013) also analyzed data obtained from different sites and observed that larger values of $C_{D}$ occur in neutral conditions. The higher values of $C_{D}$ were observed in near-neutral conditions compared to those observed at strong instability (Niu et al. 2012) for the dataset collected at a site in the Nanjing University of Information Science and Technology (Jiangsu, China).

The decreasing tendency of drag coefficient with increasing instability was also observed over the sea surface (Konishi and Nan-niti 1979; Tsukamoto et al. 1991). Konishi and Nan-Niti (1979) cited a personal communication (T. Hanabusa et al. 1976) for pointing out an observed decreasing trend with increasing instability over the land surface under the rough conditions at the Tsukuba meteorological observation tower of the Meteorological Research Institute (Japan).

Recently, Peng and Sun (2014) have also observed a decrease in $C_{D}$ with increasing instability over an urban roughness sublayer with a peak value that occurred at approximately $\zeta=-0.2$ (Peng and Sun 2014). Harman (2012) observed similar behavior of $C_{D}$ over a forest canopy with peak value at $\zeta=-0.15$ (Peng and Sun 2014).

On the other hand, a systematic mathematical analysis (appendix A) shows that $C_{D}$ increases monotonically with increasing extent of instability in the layer for similarity functions $\varphi_{m}$ and $\varphi_{h}$ as a function of $\zeta$ in the form suggested by Businger et al. (1971), Dyer (1974), and Högström (1996). These forms for similarity functions are commonly used in large-scale numerical models for computation of fluxes in unstable conditions (Yang et al. 2001; Sharan and Srivastava 2014). The analysis shows that MOS theory is consistent in weak to moderate instability, but it is unable to capture the observed decreasing behavior of $C_{D}$ with increasing instability over land surfaces in moderately to strongly unstable conditions in the framework of the commonly used similarity functions (Businger et al. 1971; Dyer 1974; Högström 1996). The mathematical analysis presented in appendix B shows that the results presented in appendix A hold well as long as $\varphi_{m}$ is continuously decreasing with increasing $-\zeta$, and this condition holds 
(a)

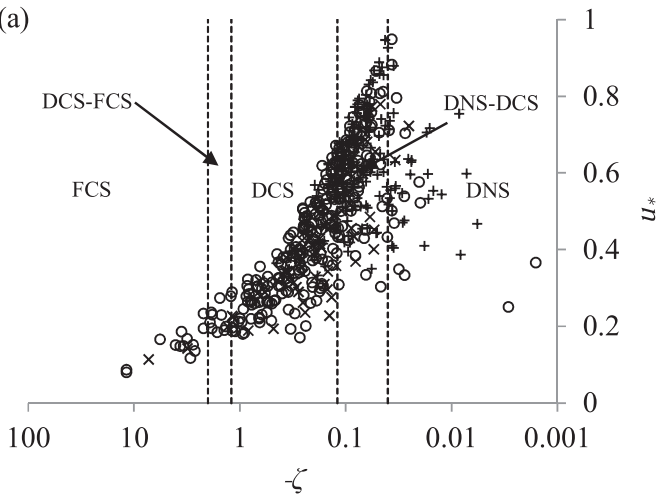

(b)

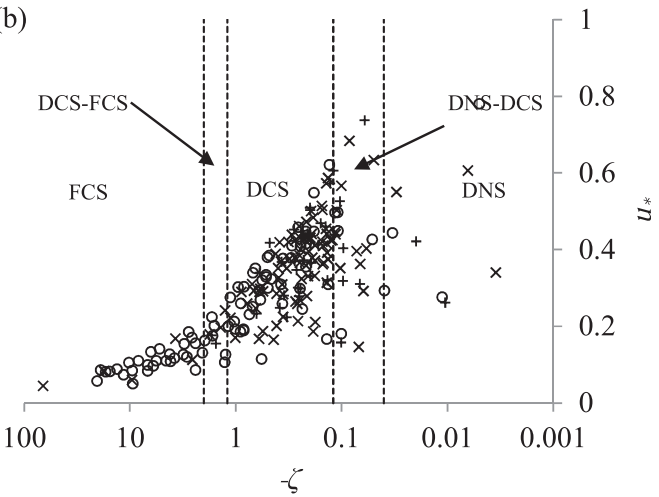

FIG. 6. Variation of $u_{*}$ with $\zeta$ in different unstable sublayers during March-May 2009. The symbols $\times, \bigcirc$, and + correspond to the data points falling in the regions I, II, and III, respectively: (a) moderate winds and (b) low winds. The vertical dashed lines separate the different sublayers.

well in all these commonly used functions. If $\varphi_{m}$ is chosen in such a way that it decreases with $-\zeta$ until $\zeta \approx \zeta_{c}$ and then starts increasing with increasing $-\zeta$ (appendix B), MOS theory can capture the observed behavior of $C_{D}$ with $\zeta$. Some of the studies reported in the literature have also speculated the increasing behavior of $\varphi_{m}$ in very unstable conditions (Kader and Yaglom 1990; Brutsaert 1992).

Rao and Narasimha (2006) also pointed out the inadequacy of MOS theory to explain the MONTBLEX90 data at Jodhpur in low-wind convective conditions and proposed a subregime of weakly forced convection within the regime of mixed convection, which is governed by velocity scales determined by the heat flux.

By analyzing the CASES-99 dataset, Vickers and Mahrt (2003) observed the decreasing nature of $C_{D}$ with increasing instability for some ranges of $-\zeta$ for the 5- and 30-min flux sets. They concluded that such a type of behavior does not seem physical and is not predicted by similarity theory. This behavior was shown to occur in the nocturnal boundary layer, where the bulk Richardson number is positive, yet the heat flux calculated using a 5- or 30-min averaging time is upward, thus falsely indicating unstable and negative $\zeta$ (Vickers and Mahrt 2003). However, we do not observe a decreasing trend in $C_{D}$ with instability from the analysis of the CASES99 data during the daytime period (0800-1600 LST). Further, based on the analysis of the Microfronts dataset (Sun 1999; Howell and Sun 1999), Mahrt et al. (2001) observed no definite trend of $C_{D}$ with the stability for $\zeta<-0.1$.

Thus, it seems that the decreasing nature of $C_{D}$ from its peak value with increasing instability might predominately appear in a tropical region. Based on the analysis of various datasets, we speculate that the decreasing nature of drag coefficient with increasing instability in a moderate- to strong-instability regime may be associated with a tropical phenomenon.

\section{Issues and limitations}

In this study, the observed behavior of drag coefficient is analyzed with respect to the wind speed and stability parameter. Some underlying issues and the limitations associated with the analysis are discussed.

\section{a. Misalignment of wind and stress vector}

In a constant flux layer, if the coordinate system is aligned with the mean wind, the stress vector should be parallel to the mean wind direction. However, a significant angle between mean wind and stress vectors is observed over sea as well as land surfaces (Geernaert 1988; Rieder et al. 1994; Weber 1999; Bernardes and Dias 2010). The misalignment of mean wind and stress vectors increases with increasing instability that might be attributed to an Ekman-layer effect of momentum transport by large eddies (Mahrt et al. 2001; Bernardes and Dias 2010). The angle $\alpha$ between mean wind and stress vectors is calculated according to Weber (1999) and shown in Fig. 7. A significantly large deviation of $\alpha$ from $0^{\circ}$ is observed, and only about $20 \%$ of data points are found between $-20^{\circ}$ and $+20^{\circ}$ degree. The values of $\alpha$ are observed to increase with increasing instability with small values in near-neutral conditions similar to the observation of Bernardes and Dias (2010). The angle seems to be dependent on $U$ as well as on $\zeta$, with small values generally associated with moderate-wind conditions. However, it is not obvious enough to analyze the effect of the misalignment on the values of $C_{D}$ in a deterministic manner. 


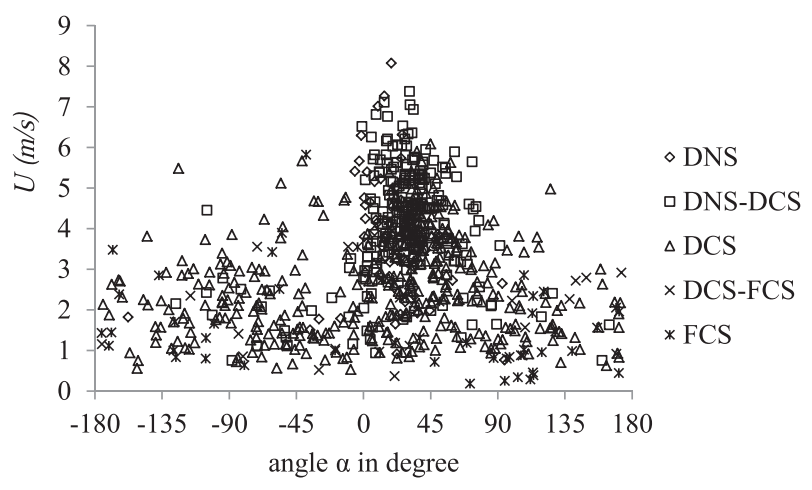

FIG. 7. Variation of $\alpha\left(^{\circ}\right)$ between stress and wind direction, with the wind speed in different unstable sublayers during MarchMay 2009.

\section{b. Nonavailability of mixed-layer height and convective velocity scale}

In the case of strong instability when the surface stress becomes small, the surface-layer scaling appears to break down; in this situation, an alternative scaling, such as either mixed-layer similarity or local free-convection similarity scaling, can be used. However, in the present study, it is not possible to use these scales because of the nonavailability of mixed-layer height $z_{i}$ and convective velocity scale $w_{*}$. The velocity scale based on the heat flux rather than frictional velocity, as suggested by Rao and Narasimha (2006), needs to be evaluated as an alternative to the MOS theory using the slow measurements along with the turbulent measurements used here.

\section{c. Self-correlation}

Self-correlation is referred to, in the literature, as spurious correlation or the shared variable problem that arises when one (dimensionless) group of variables is plotted against another and the two groups under consideration have one or more common variables (Klipp and Mahrt 2004). Although MOS theory is widely used to compute surface fluxes, physical interpretation of MOS can be ambiguous because of circular dependencies and self-correlation (Hicks 1978; Kenney 1982; Andreas and Hicks 2002; Klipp and Mahrt 2004; Baas et al. 2006; Vickers et al. 2015). In the present analysis, both $C_{D}$ and $-\zeta$ contain $u_{*}$ as a common variable, causing spurious self-correlation between $C_{D}$ and $U$ as well as between $C_{D}$ and $-\zeta$ relationships. The existence of a common variable can result in a significant self-correlation between both the relationships. To analyze the presence of self-correlation (Andreas and
Hicks 2002), we have randomized the values of $u_{*}$ while keeping the values of $U$ and $\overline{w^{\prime} \theta_{v}^{\prime}}$ fixed and recalculated the values of $C_{D}$ and $-\zeta$. This process is repeated a number of times, and the relationships of $C_{D}$ and $U$ as well as those of $C_{D}$ and $-\zeta$ are reanalyzed. The behavior appears to be similar but relatively more scattered, which suggests the presence of significant selfcorrelation in the $C_{D}$ and $U$ as well as the $C_{D}$ and $-\zeta$ relationship. However, we do not have a fundamental relationship between $C_{D}$ and $-\zeta$ (Klipp and Mahrt 2004), so we are not in a position to examine the extent of self-correlation and differentiate the physical and spurious correlations in a quantitative manner.

Mahrt (2008) also observed that the correlation between $C_{D}$ and $\zeta$ is strongly influenced by self-correlation through the shared variable $u_{*}$, and its physical significance cannot be evaluated from the data. We hasten to point out here that the analysis of the dataset suffers from the problem of self-correlation.

\section{Conclusions}

The observed behavior of the drag coefficient is analyzed with respect to wind speed and stability parameter in the different unstable sublayers from the turbulent measurements taken at Ranchi in a tropical region. The average value of $C_{D}$ is found to be almost one order larger in low winds compared with moderate-wind conditions. The variation of $C_{D}$ with $U$ is observed to follow the power-law profile. The parameterizations of $C_{D}$ with $U$ are proposed in five unstable sublayers, as suggested by Kader and Yaglom (1990) and Bernardes and Dias (2010).

The variation of $C_{D}$ with $\zeta$ is shown to be bounded by a curve. This curve first increases with $-\zeta$ until it reaches a peak at $\zeta \approx-0.12$ and then decreases with increasing instability. This trend is also observed from the turbulent measurements taken at Anand during the LASPEX field program. On the other hand, based on the analysis of MOS equations, $C_{D}$ is found to increase monotonically with increasing instability for commonly used functions (Businger et al. 1971; Dyer 1974; Högström 1996). This suggests that the MOS theory is able to capture the increasing nature of the drag coefficient in weakly to moderately unstable conditions. However, it does not explain the observed decreasing behavior of $C_{D}$ with $-\zeta$ in moderately to strongly unstable conditions in tropics within the framework of commonly used similarity functions.

Acknowledgments. The authors wish to thank Dr. Manoj Kumar for providing observational data. This work is 


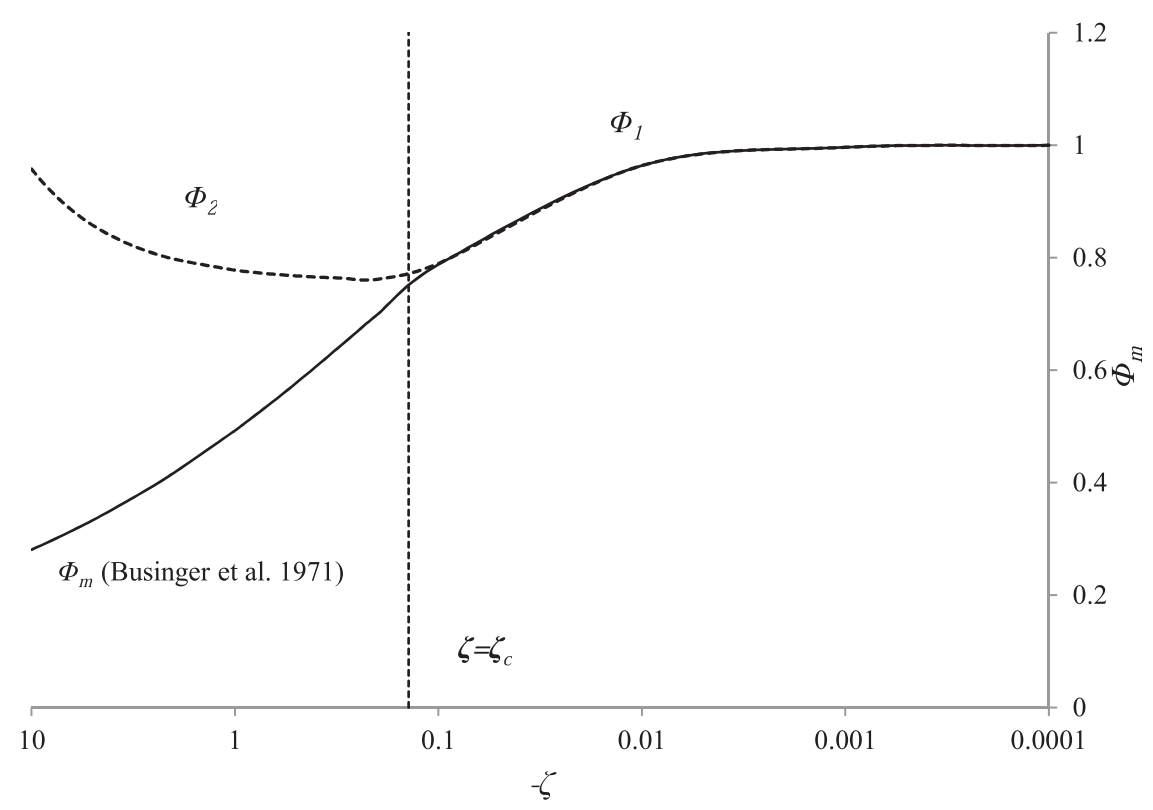

FIG. A1. Variation of $\varphi_{m}$ with $\zeta$. The vertical dotted line $\zeta=\zeta_{c}$ separates the two regions (i) $\zeta_{c} \leq \zeta \leq 0$ and (ii) $\zeta \leq \zeta_{c}$. The solid curve shows the similarity function of Businger et al. (1971), while the dashed curve shows the similarity function speculated in the present study.

partially supported by the Ministry of Earth Sciences, Government of India under the CTCZ program. We also thank Dr. Larry Mahrt for his valuable comments. The authors wish to thank the reviewers for their comments and suggestions.

\section{APPENDIX A}

\section{Mathematical Analysis of $C_{D}$ with $\zeta$}

According to MOS theory, the wind profile in a homogeneous surface layer is given as

$$
U(z)=\frac{u_{*}}{k}\left[\ln \left(\frac{z}{z_{0}}\right)-\psi_{m}\left(\zeta, \frac{z_{0}}{z} \zeta\right)\right] .
$$

The integrated similarity function $\psi_{m}$ is given by

$$
\psi_{m}\left(\zeta, \frac{z_{0}}{z} \zeta\right)=\int_{\left(z_{0} / z\right) \zeta}^{\zeta} \frac{1-\varphi_{m}(\chi)}{\chi} d \chi,
$$

in which $\chi$ is a dummy variable of integration corresponding to $\zeta$.

The similarity function $\varphi_{m}$ in unstable conditions is of the form (Businger et al. 1971):

$$
\varphi_{m}(\zeta)=\left(1-\gamma_{m} \zeta\right)^{-1 / 4}
$$

in which $\gamma_{m}$ is constant.
From Eqs. (A2) and (A3), the integrated similarity function $\psi_{m}$ can be written as

$$
\begin{aligned}
\psi_{m}\left(\zeta, \frac{z_{0}}{z} \zeta\right)= & \ln \left(\frac{1+x^{2}}{1+x_{0}^{2}}\right)+2 \ln \left(\frac{1+x}{1+x_{0}}\right) \\
& -2\left[\tan ^{-1}(x)-\tan ^{-1}\left(x_{0}\right)\right]
\end{aligned}
$$

in which $x=\left(1-\gamma_{m} \zeta\right)^{1 / 4}$ and $x_{0}=\left(1-\gamma_{m} \frac{z_{0}}{z} \zeta\right)^{1 / 4}$.
The drag coefficient $C_{D}$ is defined as

$$
C_{D}=\frac{k^{2}}{Y_{m}^{2}}
$$

in which

$$
Y_{m}=\ln \left(\frac{z}{z_{0}}\right)-\psi_{m}\left(\zeta, \frac{z_{0}}{z} \zeta\right)
$$

The increasing (decreasing) behavior of $C_{D}$ is equivalent to the decreasing (increasing) nature of the function $Y_{m}^{2}$. Thus, we examine the behavior of $Y_{m}^{2}$ with $\zeta$. For brevity, we put $\eta=-\zeta$ in Eq. (A6), implying that $\eta>0$ in unstable conditions and differentiating $Y_{m}^{2}$ with respect to $\eta$; one gets

$$
\frac{d}{d \eta}\left(Y_{m}^{2}\right)=2 Y_{m} Y_{m}^{\prime}
$$


where $Y_{m}^{\prime}$ is the derivative of $Y_{m}$ with respect to $\eta$, given as

$$
Y_{m}^{\prime}=-\left[\psi_{m}^{\prime}\left(\eta, \frac{z_{0}}{z} \eta\right)\right]
$$

Here, the prime denotes the derivative with respect to $\eta$. Applying Leibnitz's theorem (Flanders 1973) in Eq. (A2) for computing the derivative of $\psi_{m}$ with respect to $\eta, Y_{m}^{\prime}$ is given by

$$
Y_{m}^{\prime}=-\frac{1}{\eta}\left[\varphi_{m}\left(\frac{z_{0}}{z} \eta\right)-\varphi_{m}(\eta)\right]
$$

As $z_{0} / z<1, \varphi_{m}\left[\left(z_{0} / z\right) \eta\right]>\varphi_{m}(\eta)$ [Eq. (A3)], so $Y_{m}^{\prime}<0$ and $\psi_{m}^{\prime}>0$. Notice that $\psi_{m}^{\prime}>0$ for all $\eta>0$, implying that $\psi_{m}$ is a continuously increasing function of $\eta$, reaching an asymptotic value $\ln \left(z / z_{0}\right)$ as $\eta$ approaches infinity [Eq. (A4)]. This suggests that $Y_{m}$ is positive and approaches zero as $\eta=-\zeta$ is sufficiently large.

Thus, the above arguments show that (i) $Y_{m}^{\prime}<0$ and (ii) $Y_{m}>0$ for $\eta>0$, and, accordingly, $Y_{m} Y_{m}^{\prime}<0$. This implies that $Y_{m}^{2}$ is a continuously decreasing function of $\eta=-\zeta$. This is equivalent to the continuously increasing nature of $C_{D}$ with $-\zeta$.

\section{APPENDIX B}

\section{An Illustration with an Alternative Form of $\varphi_{m}$}

Notice that the results presented in appendix A hold well as long as the similarity function $\varphi_{m}$ is continuously decreasing with increasing $-\zeta$, and this condition holds well in all the commonly used functions proposed by Businger et al. (1971), Dyer (1974), and Högström (1996).

Let us consider a function $\varphi_{m}$ of the form (Fig. A1)

$$
\varphi_{m}(\zeta)=\left\{\begin{array}{ll}
\varphi_{1}(\zeta), & \zeta_{c} \leq \zeta \leq 0 \\
\varphi_{2}(\zeta), & \zeta \leq \zeta_{c}
\end{array},\right.
$$

in which $\varphi_{1}(\zeta)$ is a continuously decreasing function of $-\zeta$ in the range $\zeta_{c} \leq \zeta \leq 0$, and $\varphi_{2}(\zeta)$ is an increasing function for $\zeta \leq \zeta_{c}$. This can be interpreted that the similarity function $\varphi_{m}$ defined by Eq. (B1) decreases continuously with increasing $-\zeta$ up to $\zeta=\zeta_{c}$, beyond which it increases. Taking $\eta=-\zeta$ and $\eta_{c}=-\zeta_{c}$ as in appendix A, for this functional form of $\varphi_{m}$, Eq. (A9) shows that $Y_{m}^{\prime}<0$ and $\psi_{m}^{\prime}>0$ for $0 \leq \eta \leq \eta_{c}$, but $Y_{m}^{\prime}>0$ and $\psi_{m}^{\prime}<0$ for $\eta \geq \eta_{c}$. This suggests that $Y_{m}$ is a positive decreasing function for $0 \leq \eta \leq \eta_{c}$, and it increases with $\eta$ for $\eta \geq \eta_{c}$. Thus, $C_{D}$ increases with increasing $-\zeta$ for $\zeta_{c} \leq \zeta \leq 0$ and then decreases with increasing $-\zeta$ for $\zeta \leq \zeta_{c}$.

\section{REFERENCES}

Andreas, E. L, and B. B. Hicks, 2002: Comments on "Critical test of the validity of Monin-Obukhov similarity during convective conditions." J. Atmos. Sci., 59, 2605-2607, doi:10.1175/ 1520-0469(2002)059<2605:COCTOT >2.0.CO;2.

Baas, P., G. J. Steeneveld, B. J. H. van de Wiel, and A. A. M. Holtslag, 2006: Exploring self-correlation in flux-gradient relationships for stably stratified conditions. J. Atmos. Sci., 63 , 3045-3054, doi:10.1175/JAS3778.1.

Bernardes, M., and N. L. Dias, 2010: The alignment of the mean wind and stress vectors in the unstable surface layer. Bound.Layer Meteor., 134, 41-59, doi:10.1007/s10546-009-9429-8.

Brutsaert, W., 1992: Stability correction functions for the mean wind speed and temperature in the unstable surface layer. Geophys. Res. Lett., 19, 469-472, doi:10.1029/92GL00084.

Businger, J. A., J. C. Wyngaard, Y. Izumi, and E. F. Bradley, 1971: Flux-profile relationships in the atmospheric surface layer. J. Atmos. Sci. 28, 181-189, doi:10.1175/1520-0469(1971)028<0181: FPRITA $>2.0 . \mathrm{CO} ; 2$.

Dwivedi, A. K., S. Chandra, M. Kumar, S. Kumar, and N. V. P. K. Kumar, 2014: Spectral analysis of wind and temperature components during lightning in pre-monsoon season over Ranchi. Meteor. Atmos. Phys., 127, 95-105, doi:10.1007/s00703-014-0346-0.

Dyer, A. J., 1974: A review of flux-profile relationships. Bound.Layer Meteor., 7, 363-372, doi:10.1007/BF00240838.

Flanders, H., 1973: Differentiation under the integral sign. Amer. Math. Mon., 80, 615-627, doi:10.2307/2319163.

Furst, J. J., 2013: An investigation of vertical turbulent transport processes in coastal regions using tower observations. M. S. thesis, Dept. of Geosciences, Florida International University, 72 pp. [Available online at http://digitalcommons.fiu.edu/ etd/814.]

Geernaert, G. L., 1988: Measurements of the angle between the wind vector and wind stress vector in the surface over the North Sea. J. Geophys. Res., 93, 8215-8220, doi:10.1029/ JC093iC07p08215.

Harman, I. N., 2012: The role of roughness sublayer dynamics within surface exchange schemes. Bound.-Layer Meteor., 142, 1-20, doi:10.1007/s10546-011-9651-z.

Hicks, B. B., 1978: Some limitations of dimensional analysis and power laws. Bound.-Layer Meteor., 14, 567-569, doi:10.1007/ BF00121895.

Högström, U., 1996: Review of some basic characteristics of the atmospheric surface layer. Bound.-Layer Meteor., 78, 215-246, doi:10.1007/BF00120937.

Howell, J. F., and J. Sun, 1999: Surface-layer fluxes in stable conditions. Bound.-Layer Meteor., 90, 495-520, doi:10.1023/ A:1001788515355.

Kader, B. A., and A. M. Yaglom, 1990: Mean fields and fluctuation moments in unstably stratified turbulent boundary layers. J. Fluid Mech., 212, 637-662, doi:10.1017/S0022112090002129.

Kenney, B. C., 1982: Beware of spurious self-correlations! Water Resour. Res., 18, 1041-1048, doi:10.1029/WR018i004p01041.

Klipp, C., and L. Mahrt, 2004: Flux-gradient relationship, selfcorrelation and intermittency in the stable boundary layer. Quart. J. Roy. Meteor. Soc., 130, 2087-2103, doi:10.1256/qj.03.161.

Konishi, T., and T. Nan-niti, 1979: Observed relationship between the drag coefficient, $C d$, and stability parameter, $(-z / \mathrm{L})$. J. Oceanogr. Soc. Japan, 35, 209-214, doi:10.1007/BF02108641.

Mahrt, L., 2008: Bulk formulation of surface fluxes extended to weak-wind stable conditions. Quart. J. Roy. Meteor. Soc., $\mathbf{1 3 4}$ 1-10, doi:10.1002/qj.197. 
D. Vickers, J. Sun, N. O. Jensen, H. Jørgensen, E. Pardyjak, and H. Fernando, 2001: Determination of the surface drag coefficient. Bound.-Layer Meteor., 99, 249-276, doi:10.1023/ A:1018915228170.

Monin, A. S., and A. M. Obukhov, 1954: Basic laws of turbulent mixing in the ground layer of the atmosphere. Tr. Geofiz. Inst., Akad. Nauk SSSR, 151, 1963-1987.

Niu, S., L. Zhao, C. Lu, J. Yang, J. Wang, and W. Wang, 2012: Observational evidence for the Monin-Obukhov similarity under all stability conditions. Adv. Atmos. Sci., 29, 285-294, doi:10.1007/s00376-011-1112-6.

Patil, M. N., 2006: Aerodynamic drag coefficient and roughness length for three seasons over a tropical western Indian station. Atmos. Res., 80, 280-293, doi:10.1016/ j.atmosres.2005.10.005.

Peng, Z., and J. Sun, 2014: Characteristics of the drag coefficient in the roughness sublayer over a complex urban surface. Bound. Layer Meteor., 153, 569-580, doi:10.1007/s10546-014-9949-8.

Rao, K. G., and R. Narasimha, 2006: Heat-flux scaling for weakly forced turbulent convection in atmosphere. J. Fluid Mech., 547, 115-135, doi:10.1017/S0022112005007251.

_,$\ldots$, and A. Prabhu, 1996: Estimation of drag coefficient at low wind speeds over the monsoon trough land region during MONTBLEX-90. Geophys. Res. Lett., 23, 2617-2620, doi:10.1029/96GL02368.

Rieder, K. F., J. A. Smith, and R. A. Weller, 1994: Observed directional characteristics of the wind, wind stress, and surface waves over the open ocean. J. Geophys. Res., 99, 22 58922 596, doi:10.1029/94JC02215.

Sharan, M., and P. Kumar, 2011: Estimation of upper bounds for the applicability of nonlinear similarity functions for nondimensional wind and temperature profiles in the surface layer in very stable conditions. Proc. Roy. Soc. London, A467, 473496, doi:10.1098/rspa.2010.0220.

- , and P. Srivastava, 2014: A semi-analytical approach for parametrization of the Obukhov stability parameter in the unstable atmospheric surface layer. Bound.-Layer Meteor., 153, 339-353, doi:10.1007/s10546-014-9948-9.

Sun, J., 1999: Diurnal variations of thermal roughness height over a grassland. Bound.-Layer Meteor., 92, 407-427, doi:10.1023/ A:1002071421362.

Tsukamoto, O., E. Ohtaki, Y. Iwatani, and Y. Mitsuta, 1991: Stability dependence of drag and bulk transfer coefficients over a coastal sea surface. Bound.-Layer Meteor., 57, 359-375, doi:10.1007/BF00120054.

Tyagi, B., A. N. V. Satyanarayana, M. Kumar, and N. C. Mahanti, 2012: Surface energy and radiation budget over a tropical station: An observational study. Asia-Pac. J. Atmos. Sci., 48, 411-421, doi:10.1007/s13143-012-0037-z.

Vickers, D., and L. Mahrt, 1997: Quality control and flux sampling problems for tower and aircraft data. J. Atmos. Oceanic Technol., 14, 512-526, doi:10.1175/1520-0426(1997)014<0512: QCAFSP $>2.0 . \mathrm{CO} ; 2$.

$\longrightarrow$, and -2003 : The cospectral gap and turbulent flux calculations. J. Atmos. Oceanic Technol., 20, 660-672, doi:10.1175/ 1520-0426(2003)20<660:TCGATF $>2.0 . \mathrm{CO} ; 2$.

,,-- and E. L Andreas, 2015: Formulation of the sea surface friction velocity in terms of the mean wind and bulk stability. J. Appl. Meteor. Climatol., 54, 691-703, doi:10.1175/ JAMC-D-14-0099.1.

Weber, R. O., 1999: Remarks on the definition and estimation of frictional velocity. Bound.-Layer Meteor., 93, 197-209, doi:10.1023/A:1002043826623.

Yang, K., N. Tamai, and T. Koike, 2001: Analytical solution of surface layer similarity equations. J. Appl. Meteor., 40, 1647-1653, doi:10.1175/1520-0450(2001)040<1647:ASOSLS > 2.0.CO;2.

Yusup, Y., 2012: Aerodynamic drag coefficient over equatorial coastal industrialized and urban areas. J. Wind Eng. Ind. Aerodyn., 110, 25-39, doi:10.1016/j.jweia.2012.07.006.

Zhu, P., and J. Furst, 2013: On the parameterization of surface momentum transport via drag coefficient in low-wind conditions. Geophys. Res. Lett., 40, 2824-2828, doi:10.1002/grl.50518. 